\title{
Tratamiento farmacológico de COVID-19: Conferencia de la Peruvian American Medical Society (PAMS)
}

\author{
The pharmacological treatment of COVID-19: \\ Conference of Peruvian American Medical Society (PAMS) \\ Jose Armando Gonzales-Zamora
}

\section{Resumen}

El tratamiento farmacológico de COVID-19 ha cambiado mucho durante la pandemia. Medicamentos como la hidroxicloroquina dejaron de utilizarse ya que se demostró que carecían de eficacia clínica. Ivermectina, un medicamento empleado mucho en Perú, carece de evidencia científica suficiente que permita su recomendación. A la fecha, el tratamiento de pacientes hospitalizados se basa en Remdesivir y corticoides; y el de pacientes ambulatorios es solo sintomático, no recomendándose tampoco el uso empírico de antibióticos. El uso de Tocilizumab es todavía controversial ya que los resultados de los ensayos clínicos son contradictorias. A pesar de la frecuencia más alta de eventos trombóticos en COVID-19, no se recomienda en la actualidad la anticoagulación terapéutica empírica.

Palabras clave: COVID-19, tratamiento, Remdesivir, corticoides, Tocilizumab, anticoagulación.

\section{Abstract}

The pharmacological treatment of COVID-19 has changed a lot during the pandemic. Hydroxychloroquine is not :d anymore due to the lack of clinical efficacy. Ivermectin, a medication applied widely in Peru, is not recommended oecause the scientific evidence to support its use is insufficient. To date, the treatment of hospitalized patients is based in Remdesivir and corticosteroids, and it is only symptomatic without empiric antibiotics in the ambulatory setting. The use of Tocilizumab is still controversial due to the contradictory results of the clinical trials. Although there is probably a higher frequency of thrombotic events in COVID-19, therapeutic anticoagulation is not recommended.

Keywords: COVID-19, treatment, Remdesivir, corticosteroids, Tocilizumab, anticoagulation.

La Peruvian American Medical Society (PAMS) realizó el 17 y 18 de setiembre de 2020 una conferencia virtual sobre COVID-19, contando con varias sesiones enfocadas a revisar las principales afecciones en distintos órganos producidas por SARS-CoV-2; además se conversó sobre los tratamientos para COVID-19, incluyendo el uso de drogas antivirales, plasma del convaleciente y el desarrollo de las vacunas contra este mal. Por intermedio de esta carta, presento los datos expuestos en la charla sobre Manejo de COVID-19 llevada a cabo el 18 de setiembre.

El tratamiento farmacológico de COVID-19 ha evolucionado mucho durante la pandemia. Inicialmente el estudio francés llevado a cabo por Gautret P. et al. nos dio la esperanza sobre la utilidad de la hidroxicloroquina para el tratamiento de COVID-19, ya que su estudio nos mostraba una negativización más rápida de la carga viral producida por SARS-CoV-2 en pacientes que recibieron hidroxicloroquina mas Azitromicina ${ }^{(1)}$. Sin embargo, este dato alentador se convirtió rápidamente en una total decepción, ya que ensayos clínicos posteriores demostraron que la hidroxicloroquina no tenía ningún beneficio clínico en pacientes infectados por COVID-19 en ningún estadio de la enfermedad, y a la fecha su uso no se recomienda en pacientes hospitalizados o ambulatorios $^{(2-4)}$. Una de las prácticas que se hizo muy popular en Perú,

${ }^{1}$ Médico Infectológo, MD. Division of Infectious Diseases. Department of Medicine. University of Miami, Miller School of Medicine. Miami, Florida. USA. Peruvian American Medicine Society (PAMS). USA. ORCID: https://orcid.org/0000-0002-2768-9712 
fue el uso de ivermectina para tratamiento de COVID. Esta indicación todavía figura en la norma técnica nacional del Ministerio de Salud de Perú (MINSA); sin embargo, carece de suficiente fundamento científico, ya que la evidencia es muy limitada y se circunscribe principalmente a un estudio australiano que mostró que la ivermectina inhibía la replicación viral in-vitro ${ }^{(5,6)}$. Además, existen algunos estudios en su mayoría observacionales retrospectivos que muestran cierto beneficio; no pudiéndose extrapolar directamente a la práctica clínica debido a limitaciones inherentes a este tipo de estudios $^{(7)}$. Todo lo mencionado hace que la ivermectina no sea un medicamento recomendado para el tratamiento de COVID en ninguna de sus fases, ni en pacientes hospitalizados y tampoco en ambulatorios. Esta recomendación es compartida por muchas sociedades científicas tanto de USA como Latinoamérica $^{(8)}$.

Con respecto al uso de antibióticos, la evidencia actual nos muestra que la frecuencia de coinfección bacteriana en COVID es baja, alrededor del 7\%.9 Los patógenos bacterianos más comúnmente reportados son Mycoplasma pneumoniae, Pseudomona aeruginosa, Haemophilus influenzae y Klebsiella pneumoniae $^{(9)}$. A pesar de que Streptococcus pneumoniae constituye uno de los principales patógenos asociados con el virus de la influenza, su ocurrencia es muy rara en el contexto de la infección por SARS-CoV-2. Asimismo, el estudio publicado por Buetti N. et al. nos muestra que la administración temprana de antibióticos no impacta la mortalidad de pacientes con COVID-19 y tampoco retrasa el desarrollo de infecciones en pacientes críticos $^{(10)}$. Todo ello nos lleva a concluir que el uso de antibióticos no está recomendado en pacientes con COVID provenientes de la comunidad.

A la fecha, el único antiviral recomendado para el tratamiento de COVID es el Remdesivir, el cual inhibe la ARN polimerasa del SARS-CoV-2 y produce una terminación prematura de la replicación viral. Este medicamento cuenta con la aprobación de la FDA para el tratamiento de pacientes hospitalizados con COVID-19. Su uso se fundamenta principalmente en el ensayo clínico ACTT-1, que demostró una reducción significativa de 5 días en el tiempo de recuperación al ser comparado con placebo ${ }^{(11)}$. Asimismo, el estudio ACTT-1 mostro una reducción numérica de la mortalidad que no alcanzo significancia estadística ${ }^{(11)}$. Remdesivir constituye actualmente la terapia de primera línea para COVID en pacientes hospitalizados, y su uso está recomendado por muchas guías internacionales incluida la del Instituto Nacional de Salud Americano $(\mathrm{NIH})^{(8)}$. Recientemente el estudio SOLIDARITY publicó sus resultados (aún no revisados por pares) que mostraban una carencia de beneficio clínico con el uso de Remdesivir, lo cual contradice los hallazgos anteriormente descritos ${ }^{(12)}$. Sin embargo, hay que destacar que la calidad de evidencia del estudio SOLIDARITY es inferior al ACTT-1, ya que se trata de un estudio de etiqueta abierta no controlado con placebo, en el cual no se estratifica las características clínicas basales funcionales de los pacientes. El estudio SOLIDARITY se realiza principalmente en países del tercer mundo, contando con muchos centros de investigación con capacidad muy heterogénea para brindar tratamiento de soporte, lo cual puede afectar el desenlace de los pacientes tratados. Hay que resaltar también que la duración del tratamiento con Remdesivir en el estudio SOLIDARITY fue de 10 días, lo cual contribuye a un sesgo en contra de la droga de estudio, ya que, al ser una droga endovenosa, los pacientes tenían que permanecer hospitalizados, lo cual hace difícil evaluar la reducción de la estancia hospitalaria de pacientes $\operatorname{tratados}^{(12)}$

Al momento de la publicación de este artículo, el único medicamento que ha demostrado una reducción significativa de la mortalidad a los 28 días son los corticoides, y una reducción muy importante, que fue evidenciada por el estudio RECOVERY, el cual mostró una disminución de 36\% de la mortalidad en pacientes en ventilación mecánica y un $18 \%$ de reducción en pacientes tributarios de oxígeno suplementario ${ }^{(13)}$. Un hallazgo a resaltar es la tendencia a incrementar la mortalidad en pacientes que no usaban suplemento de oxígeno, lo que hace que los corticoides solo se recomienden en el ámbito hospitalario en pacientes con baja saturación de oxígeno. Estos hallazgos han sido replicados por otros estudios, lo que conllevó a la realización de un meta-análisis de 7 ensayos clínicos encontrándose una reducción global de la mortalidad del $20 \%{ }^{(14)}$. Por otro lado, el tratamiento de pacientes con enfermedad leve o moderada que no utilizan oxígeno se limita solo a medicamentos sintomáticos.

Es preciso mencionar que la evolución natural de la infección por COVID consta de 3 fases, una primera fase viral, una fase pulmonar y una fase sistémica. En estas dos últimas fases se produce una reacción inflamatoria inducida por el virus, que puede llegar a comportarse como una tormenta de citoquinas. Por esta razón, uno de los tratamientos estipulados ha sido el Tocilizumab, el cual es un inhibidor de la interleukina-6, que bloquea la respuesta inflamatoria. Actualmente, la evidencia clínica de su uso es contradictoria, con algunos ensayos que no muestran beneficio clínico (COVACTA), y otros como el ensayo EMPACTA que han tenido resultados favorables, como la reducción de la 
probabilidad de progresar a ventilación mecánica o muerte en pacientes que reciben Tocilizumab en comparación con los que reciben placebo ${ }^{(15,16)}$.

A pesar del reporte creciente de eventos trombóticos en pacientes con COVID-19, el uso de anticoagulación terapéutica empírica no está recomendada ${ }^{(8)}$. Al momento, solo la tromboprofilaxis con dosis usuales de heparina o enoxaparina constituyen las medidas más aceptadas en práctica clínica, reservándose la anticoagulación terapéutica en casos confirmados de trombosis, o en aquellos en los que hay una sospecha clínica muy alta y no se tiene herramientas diagnósticas que permitan un diagnóstico de trombosis definitivo $^{(8)}$.

Existen otras terapias que se encuentran en ensayo clínico, entre las que podemos mencionar: el plasma del convaleciente, las células mesenquimales, el Aviptadil, el intercambio de plasma, el óxido nítrico inhalado, etc. ${ }^{(8)}$.

El tratamiento de COVID se encuentra en desarrollo y está sujeto a cambio constante. Sin embargo, podemos concluir basados en ensayos clínicos, que la hidroxicloroquina no debe utilizarse para el tratamiento de COVID-19 en ninguna de sus fases. Además, debido a la carencia de evidencia científica sólida, no se recomienda el uso de ivermectina en pacientes hospitalizados y tampoco en ambulatorios. Actualmente los tratamientos de primera línea son Remdesivir y corticoides, dichos tratamientos están indicados en pacientes hospitalizados, mas no en pacientes ambulatorios sin problemas de oxigenación. Finalmente, Tocilizumab y la anticoagulación terapéutica no deben utilizar de forma general ya que la evidencia actual no respalda su uso, por lo que deberían ofrecerse solo en el contexto de ensayo clínico.

\section{Referencias bibliográficas}

1. Gautret P, Lagier JC, Parola P, Hoang VT, Meddeb L, Mailhe M, et al. Hydroxychloroquine and azithromycin as a treatment of COVID-19: results of an open-label nonrandomized clinical trial. Int $\mathrm{J}$ Antimicrob Agents. 2020;56(1):105949.

2. Cavalcanti AB, Zampieri FG, Rosa RG, Azevedo LCP, Veiga VC, Avezum A, et al. Hydroxychloroquine with or without Azithromycin in Mild-to-Moderate Covid-19. N Engl J Med. 2020 19;383(21):2041-2052.

3. RECOVERY Collaborative Group, Horby P, Mafham M, Linsell L, Bell JL, Staplin N, et al. Effect of Hydroxychloroquine in Hospitalized Patients with Covid-19. NEngl J Med. 2020 Nov 19;383(21):2030-2040.

4. Skipper CP, Pastick KA, Engen NW, Bangdiwala AS, Abassi M, Lofgren SM, et al. Hydroxychloroquine in Nonhospitalized Adults With Early COVID-19 : A Randomized Trial. Ann Intern Med. 2020;173(8):623-631.

5. Manejo ambulatorio de personas afectadas por la COVID19 en el Perú, que como Anexo forma parte integrante de la presente resolución Ministerial. Ministerio de Salud (MINSA). [Citado el 24 de noviembre del 2020]. Disponible en: https://cdn.www.gob.pe/uploads/document//file/1458478/ R.M\%20N\%C2\%B0974-2020-MINSA.pdf.pdf

6. Caly L, Druce JD, Catton MG, Jans DA, Wagstaff KM. The FDA-approved drug ivermectin inhibits the replication of SARS-CoV-2 in vitro. Antiviral Res. 2020; 178:104787.

7. Rajter JC, Sherman MS, Fatteh N, Vogel F, Sacks J, Rajter JJ. Use of Ivermectin Is Associated With Lower Mortality in Hospitalized Patients With Coronavirus Disease 2019: The ICON Study. Chest. 2020 Oct 13: S0012-3692(20)34898-4.

8. Coronavirus Disease (COVID-19) Treatment guidelines. NIH. [Citado el 22 de noviembre 2020]. Disponible en: https://www.covid19treatmentguidelines.nih.gov/

9. Lansbury L, Lim B, Baskaran V, Lim WS. Co-infections in people with COVID-19: a systematic review and metaanalysis. J Infect. 2020;81(2):266-275.
10. Buetti N, Mazzuchelli T, Lo Priore E, Balmelli C, Llamas M, Pallanza M, et al. Early administered antibiotics do not impact mortality in critically ill patients with COVID-19. J Infect. 2020;81(2):e148-e149.

11. Beigel JH, Tomashek KM, Dodd LE, Mehta AK, Zingman BS, Kalil AC, et al. Remdesivir for the Treatment of Covid-19 Final Report. N Engl J Med. 2020;383(19):1813-1826.

12. WHO Solidarity trial consortium, Pan H, Peto R, Quarraisha Abdool K, Alejandria M, Henao-Restrepo AM, et al. Repurposed antiviral drugs for COVID-19-interim WHO SOLIDARITY trial results. Preprint. [Citado el 24 de noviembre del 2020]. Disponible en: https://www.medrxiv.org/ content/10.1101/2020.10.15.20209817v1

13. RECOVERY Collaborative Group, Horby P, Lim WS, Emberson JR, Mafham M, Bell JL, et al. Dexamethasone in Hospitalized Patients with Covid-19 - Preliminary Report. N Eng1 J Med. 2020 Jul 17: NEJMoa2021436.

14. WHO Rapid Evidence Appraisal for COVID-19 Therapies (REACT) Working Group, Sterne JAC, Murthy S, Diaz JV, Slutsky AS, Villar J, Angus DC, et al. Association Between Administration of Systemic Corticosteroids and Mortality Among Critically Ill Patients With COVID-19: A Metaanalysis. JAMA. 2020;324(13):1330-1341.

15. Roche provides an update on the phase III COVACTA trial of Actemra/RoActemra in hospitalised patients with severe COVID-19 associated pneumonia. Roche. Press Release. [Citado el 24 de noviembre del 2020]. Disponible en: https://www.roche.com/investors/updates/inv-update-202007-29.htm

16. Roche's phase III EMPACTA study showed Actemra/RoActemra reduced the likelihood of needing mechanical ventilation in hospitalised patients with COVID-19 associated pneumonia. Roche. Press Release. [Citado el 24 de noviembre del 2020]. Disponible en: https://www.roche.com/media/releases/med-cor-2020-0918.htm 
Contribución de autoría: Jose Armando Gonzales-Zamora ha sido el autor del estudio, contribuyendo a su concepción, búsqueda electrónica, revisión inicial, el diseño de estudio, redacción, y revisión final.

Conflicto de interés: El autor no tiene conflictos de interés con la publicación de este trabajo.

Financiamiento: Autofinanciado.

Citar como: Gonzales-Zamora, JA. Tratamiento farmacológico de COVID-19: Conferencia de la Peruvian American Medical Society (PAMS). Diagnóstico(Lima). 2020;59(3):141-144.

DOI: 10.33734/diagnostico.v59i3.237

Correspondencia: JoseA. Gonzales Zamora. Correo electrónico:jxg1416@med.miami.edu

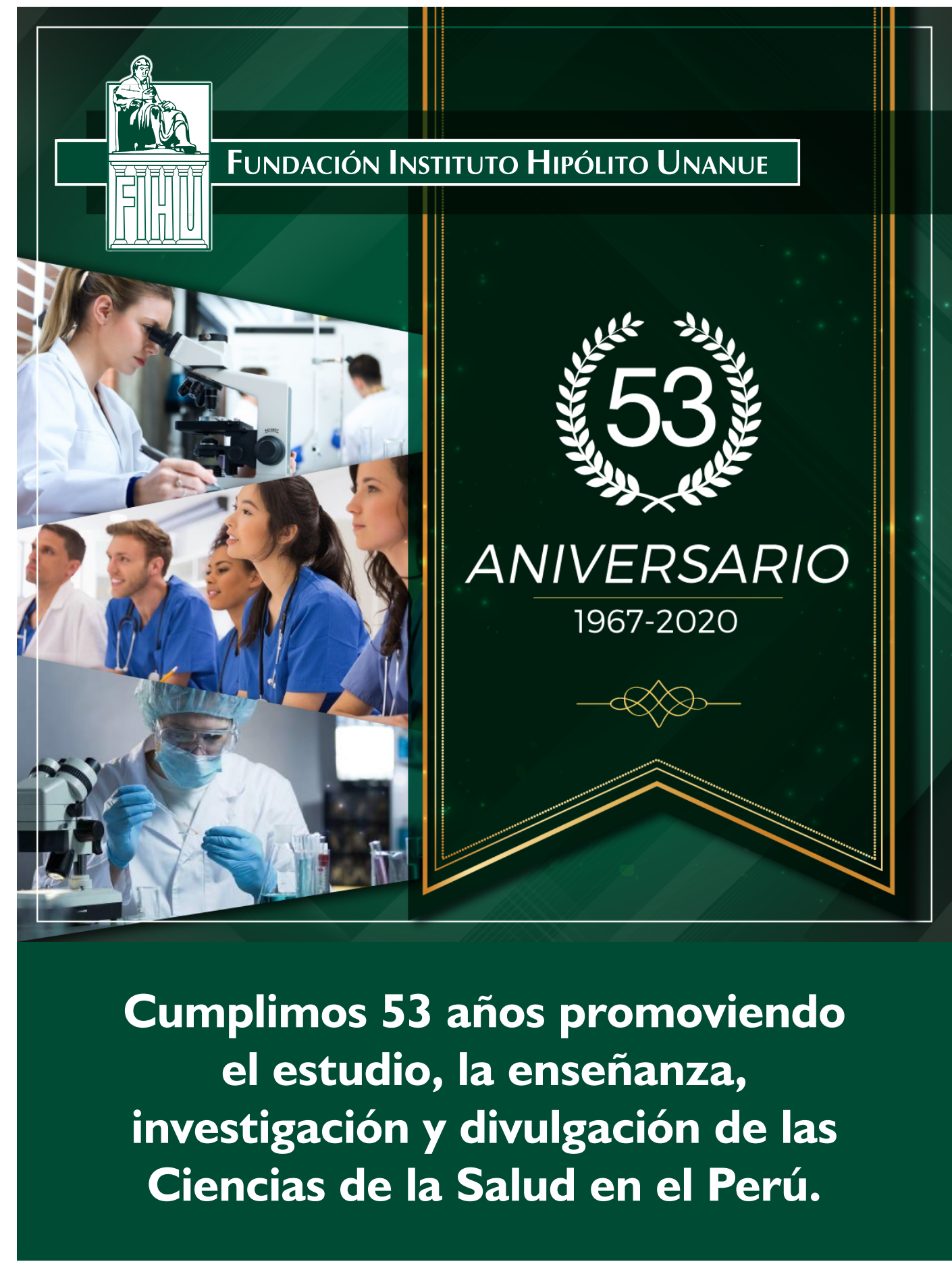

